\title{
Time, change, and motion: The effects of stimulus movement on temporal perception
}

\author{
SCOTT W. BROWN \\ University of Southerm Maine, Portland, Maine
}

\begin{abstract}
The effects of stimulus motion on time perception were examined in five experiments. Subjects judged the durations (6-18 sec) of a series of computer-generated visual displays comprised of varying numbers of simple geometrical forms. In Experiment 1, subjects reproduced the duration of displays consisting of stationary or moving (at $20 \mathrm{~cm} / \mathrm{sec}$ ) stimulus figures. In Experiment 2, subjects reproduced the durations of stimuli that were either stationary, moving slowly (at $10 \mathrm{~cm} / \mathrm{sec}$ ), or moving fast (at $30 \mathrm{~cm} / \mathrm{sec}$ ). In Experiment 3, subjects used the production method to generate specified durations for stationary, slow, and fast displays. In Experiments 4 and 5, subjects reproduced the duration of stimuli that moved at speeds ranging from 0 to $45 \mathrm{~cm} / \mathrm{sec}$. Each experiment showed that stimulus motion lengthened perceived time. In general, faster speeds lengthened perceived time to a greater degree than slower speeds. Varying the number of stimuli appearing in the displays had only limited effects on time judgments. Other findings indicated that shorter intervals tended to be overestimated and longer intervals underestimated (Vierordt's law), an effect which applied to both stationary and moving stimuli. The results support a change model of perceived time, which maintains that intervals associated with more changes are perceived to be longer than intervals with fewer changes.
\end{abstract}

The perception of time is the perception of events. Lacking "temporal receptors" which respond to "temporal stimuli," we experience time by perceiving changes in the number, magnitude, and salience of the events in an interval (Poynter, 1989). The role of stimulus events in timing has been the focus of much theoretical work (see Block, 1990; Poynter, 1989, for reviews). According to the storage-size model (Ornstein, 1969), perceived time is a function of the amount of "memory space" needed to encode and store stimulus events. More complex or numerous events require more storage space and thereby lengthen perceived time. This model has been used to explain such phenomena as the filled-duration illusion (see Fraisse, 1963), in which intervals containing more stimuli tend to be judged as longer than intervals containing fewer stimuli. However, some tests of the model have yielded contradictory or equivocal results (e.g., Block, 1978; Jankowitz, 1977; Mo, 1974; Schiffman \& Bobko, 1977), and its status remains controversial (Block,

This project was supported by a College of Arts and Sciences Summer Faculty Fellowship award and a Faculty Senate Research Grant, both provided by the University of Southern Maine. I gratefully acknowledge the contribution of Karlee S. Hoecker, Debra L. KnightAxelsen, and Shane G. May, who recruited subjects and collected data. Special thanks are extended to Judith C. Merrill, Greta Mesta, and Thomas Casset for their services in translating foreign-language publications. I am indebted to three anonymous reviewers who offered many valuable suggestions which greatly improved this paper. Requests for reprints should be sent to Scott W. Brown, Department of Psychology, University of Southern Maine, Portland, ME 04103 (e-mail: swbrown@usm.maine.edu).
1990). An alternative approach, advanced by a number of theorists, emphasizes the role of stimulus change in time perception (e.g., Block, 1982, 1990; Fraisse, 1963, 1978; Gibson, 1975; Poynter \& Homa, 1983). According to this view, changes in stimulus events lengthen the experience of time because "change is the psychological index of time passage" (Poynter, 1989, p. 309). Poynter has outlined the main features of a change/segmentation model of timing, which stresses the role of event organization in perceived time. Changing events which occur in regular, ordered sequences serve as effective temporal cues. An important factor is the extent to which a pattern of stimulus events can be subdivided into distinctive segments or chunks. The greater the segmentation of the interval, the greater the number of perceived changes and the longer the apparent duration. Some theorists (e.g., Gibson, 1975; Poynter, 1989) describe stimulus motion as an important temporal cue, and predict that moving stimuli should lengthen perceived time. In Poynter's words, "Perceived duration ... is determined by the amount of change experienced, and one salient type of change is a change in spatial location" (p. 326). By definition, moving stimuli occupy a sequence of different spatial positions, and thus provide to the observer a dynamic pattern of changing events. Further, faster speeds produce more rapid changes in spatial location than slower speeds, and so should lengthen perceived time to a greater degree. A lengthening in the perceived duration of moving objects would also be consistent with the storage-size model if it is assumed that moving stimuli are more complex than stationary stimuli, and thus require more storage space in memory. 


\section{TIME AND MOTION STUDIES}

A growing body of literature exists on the relation between time and motion, and includes such topics as velocity estimation (e.g., Algom \& Cohen-Raz, 1984; Lappin, Bell, Harm, \& Kottas, 1975; Tynan \& Sekuler, 1982), time-of-arrival estimation (e.g., Cavallo \& Laurent, 1988; Peterken, Brown, \& Bowman, 1991; Schiff \& Oldak, 1990), and motion detection (e.g., van Doorn \& Koenderink, 1984; Verri, Girosi, \& Torre, 1990). A number of studies have been concerned specifically with the perceived duration of moving stimuli. This research is centered upon two main issues, and the findings are mixed. The first issue concerns differences in the perceived duration of stationary versus moving stimuli. In a classic set of studies, Roelofs and Zeeman (1951) presented pairs of short-duration stimuli $(0.2-3.2 \mathrm{sec})$ for comparative time judgments. The stimuli were both stationary and moving (at $7.5-60 \mathrm{~cm} / \mathrm{sec}$ ) squares of light projected on a screen; subjects judged which member of each pair was displayed for a longer duration. The moving stimuli tended to be judged as lasting longer than stationary stimuli. Similar results were reported by Goldstone and Lhamon (1974), whose subjects judged the relative durations $(0.7-1.3 \mathrm{sec})$ of pairs of stimuli produced on an oscilloscope screen. The stimuli consisted of either stationary versus moving wave patterns (Experiment 2) or stationary versus rotating trapezoidal figures (Experiment 3 ). In a follow-up study (Lhamon \& Goldstone, 1975, Experiment 1), the stimuli were either stationary or moving grid patterns. In each of these studies, the moving displays were judged to be longer in duration than the stationary displays. Other findings, however, complicate this seemingly straightforward relationship between stimulus motion and perceived time. Tayama and Aiba (1982, Experiment 2) had subjects reproduce the duration $(2-16 \mathrm{sec})$ of both stationary and rotating (at $2^{\circ}, 8^{\circ}$, or $16^{\circ}$ of visual angle per sec) dot patterns displayed on a computer monitor. Although the moving displays were associated with longer reproductions, the effect diminished for the stimuli of longer duration. Tayama and his colleagues also found that the slowest moving stimuli in a set were judged to be shorter in duration than stationary stimuli (Tayama \& Aiba, 1982, Experiments 1 and 3; Tayama, Nakamura, \& Aiba, 1987 , Experiments 1 and 2). However, patterns rotating at faster speeds did show the expected result of being judged longer than stationary patterns.

The second issue involves the effect of stimulus speed on perceived time. Several experiments indicate that faster moving stimuli lead to progressively longer temporal judgments (Fraisse, 1962, Experiment 4; Leiser, Stern, \& Meyer, 1991, Experiment 2; Piaget, 1961/1969, pp. 274-277; Tayama \& Aiba, 1982, Experiment 4; Tayama et al., 1987, Experiments 1 and 2). These studies involve a variety of stimuli, durations, and speeds (up to $90 \mathrm{~cm} / \mathrm{sec}$ ). Other research suggests that the effect of stimulus speed may be influenced or moderated by various stimulus- and subject-related characteristics. The research by Roelofs and Zeeman (1951) contains numerous apparent interactions between stimulus speed, duration, distance traveled, and presentation order; unfortunately, however, the data analysis is rather sketchy and these effects are difficult to evaluate. Matsuda (1974, Experiment 2) obtained significant fourth- and fifth-order interactions involving speed, distance, trials, sex, and age. Fraisse (1962, Experiment 3 ) found that the progressive temporal lengthening effect of stimulus speed (from 10 to $30 \mathrm{~cm} / \mathrm{sec}$ ) was greater for $10 \mathrm{sec}$ than it was for $5 \mathrm{sec}$ stimulus durations. Note that this result seems to contradict that described earlier of Tayama and Aiba (1982, Experiment 2), who found that the effect of motion was weaker for stimuli of longer duration.

Further contradictions appear in studies that have found no effect of stimulus speed on time judgments (Bonnet, 1965, Experiment 1; Bonnet, 1967, Experiment 1; Bonnet, 1968, Experiment 2; Fraisse, 1962, Experiments 1 and 2). This failure to obtain the expected result has been attributed to one of two possibilitiesthat the stimulus speeds were too fast (Fraisse, 1962), or that the durations were too short (Bonnet, 1965). However, the range of speeds $(3.63-30 \mathrm{~cm} / \mathrm{sec})$ and durations $(0.32-10 \mathrm{sec})$ are similar to those used in research showing a positive relation between stimulus speed and perceived time. One notable feature is that most of these studies involve small $n$ s (typically 12 subjects or fewer), so that individual variability may have masked the effect (Bonnet, 1968). In some studies, faster stimuli are associated with shorter temporal judgments (Bonnet, 1965, Experiment 2; Brown, 1931; Matsuda, 1974, Experiments 1 and 2). Matsuda (1974) has referred to this result as the $i^{\prime}$ effect, which she regards as an opposite counterpart to the kappa effect (a lengthening of the perceived time separating two sequential stimuli when the distance between them is lengthened). Matsuda (1974) and Bonnet (1967) have speculated that different temporal cues may exert different effects on time judgments. The suggestion is that if one relies on the distance an object moves, time judgments are lengthened; if one focuses instead on the speed at which an object moves, time judgments are shortened. However, this hypothesis is challenged by research showing that both speed and distance can lengthen perceived time (Bonnet, 1968, Experiment 1; Rachlin, 1966).

Thus, the effects of stimulus motion on perceived time have not been clearly established. Although moving stimuli generally lengthen temporal judgments, and faster movement tends to lengthen judgments to a greater degree than slower movement, the literature contains numerous discrepancies, interactions, and null results. One serious difficulty in comparing these studies is that they differ widely in their scope and technical sophistication. A standardized methodology has not been established, and at least some of the inconsistencies may be due to the many methodological and procedural differences which exist between studies, such as: (1) different types of stimuli and depictions of motion (ranging from paper figures pasted onto a rotating drum to patches 
of light projected on a screen to animated forms displayed on a computer monitor); (2) various kinds of temporal judgments (including both relative and absolute judgments of time); and (3) a wide assortment of stimulus speeds and durations. Some or all of these factors may contribute to the inconsistent results.

\section{THE PRESENT RESEARCH}

The purpose of the present investigation is to clarify and extend the findings on stimulus motion and perceived time. The primary goal is to determine whether stimulus movement reliably lengthens temporal judgments. To be convincing, the effect should be repeatable across independent experiments and demonstrable with different time-judgment methods. A related goal is to determine whether increases in stimulus speed exaggerate the temporal lengthening effect, as event-based models of timing predict. The research is also designed to clarify the conflicting findings concerning the effect of the duration of moving stimuli on perceived time. Finally, the number of stimuli was manipulated to test the differing predictions of the storage-size and change/segmentation models. These points are discussed below.

The research consisted of five interrelated experiments, all employing the same general methodology. The subjects $(n=57-114)$ made absolute time judgments (reproductions or productions) of stationary and moving stimuli which were generated by computer graphics software and presented on a high-resolution CRT monitor screen. Special care was taken to control for the following important features of the experimental situation.

1. Given the interdependence of speed, distance, and duration, the manner in which stimulus movement is depicted is an important practical issue. In most previous studies, stimuli have moved either in a straight-line fashion or in a simple repetitive pattern. One serious problem with linear trajectories is that they may bias subjects to rely primarily on the distance traveled when making their time judgments, particularly if the stimuli always start from the same point (see Bonnet, 1968, Experiment 2; Rachlin, 1966, pp. 80-82). Similarly, stimuli moving continuously along a simple circular pathway (or which are briefly exposed as they pass a narrow aperture) may encourage subjects to rely on a count of the movement cycles or repetitions. Such strategies were discouraged in the present experiments by having multiple stimuli travel along a lengthy and complex nonlinear route from different starting positions on each trial.

2. A second issue concerns speed. Stimulus speeds (usually defined in terms of $\mathrm{cm} / \mathrm{sec}$ or degrees/sec) are not specified in some studies (e.g., Goldstone \& Lhamon, 1974; Leiser et al., 1991). Surprisingly, many studies lack a zero speed condition (e.g., Bonnet, 1965, 1967, 1968; Brown, 1931; Fraisse, 1962; Matsuda, 1974; Piaget, 1961/1969). Four experiments in the present series (Experiments 2, 3, 4, and 5) examined the ef- fect of speed on time judgments. The speeds ranged from 5 to $45 \mathrm{~cm} / \mathrm{sec}$, with each speed separated by at least $10 \mathrm{~cm} / \mathrm{sec}$ (and in some cases by $20 \mathrm{~cm} / \mathrm{sec}$ ). All experiments included a stationary (control) condition for comparison purposes.

3. Most prior studies have employed a restricted range of stimulus durations (often under $5 \mathrm{sec}$ ). But some studies (e.g., Fraisse, 1962; Roelofs \& Zeeman, 1951; Tayama \& Aiba, 1982) suggest that duration is an influential factor which may either enhance or reduce any temporallengthening effect of stimulus motion. The experiments reported here employed a relatively broad range of durations $(6-18 \mathrm{sec})$ as a check on the generalizability of the findings.

4. A final consideration is that subjects in previous studies typically judged the durations of single stimuli (or a unified group of smaller elements forming a larger pattern). This is an important issue from a theoretical standpoint. The storage-size model proposes that more stimuli use up more memory storage space and lengthen perceived time (the classic filled-duration illusion). According to the change/segmentation model, however, more stimulus events do not always correspond to more perceived changes and longer time judgments. Poynter (1989) argues that stimulus events that are more numerous will not lengthen perceived time unless the events form highly discrete and distinctive chunks. If, on the other hand, the events are relatively undifferentiated and lack discreteness, additional stimuli will have no effect. The same principle would presumably apply to moving objects. The implication here is that greater numbers of moving stimuli would lead to a further lengthening of perceived time only if the stimuli were highly distinctive, perhaps traveling at different speeds on identifiably different routes. In contrast, multiple stimuli of the same size and shape which move along the same route and at the same speed would be less distinctive. For example, a flock of birds in flight is perceived to be a single entity rather than comprised of separate individuals (the gestalt principle of "common fate"; see Wertheimer, 1923/ 1958). Greater numbers of such stimuli would not be expected to create any greater impression of change. The only data involving different numbers of moving stimuli are from Piaget (1961/1969, pp. 274-277), who had subjects judge the relative durations of displays consisting of small beads moving along a linear track at fast $(80 \mathrm{~cm} / \mathrm{sec})$ or slow $(40 \mathrm{~cm} / \mathrm{sec})$ speeds. He found that the fast displays were judged to be longer in duration, regardless of whether the number of beads in the fast displays was greater than or the same as that of the slow displays. The issue, however, remains essentially unexplored. In the present research (Experiments 1, 2, 3, and 5 ), the number of stimuli comprising the displays for temporal judgments was systematically varied. These stimuli were not uniquely distinctive figures moving in individualized trajectories, but rather were multiple copies of the same figure which moved along the same pathway at the same speed. According to the storagesize model, greater numbers of stimuli will lengthen 
time judgments; according to a change model, the number of stimuli in this case should have no effect.

\section{EXPERIMENT 1}

The main purpose of Experiment 1 was to test the effects of stimulus motion on perceived time. Subjects were presented with a series of stationary and moving stimulus displays and reproduced the duration of each display. It was predicted that temporal reproductions would be longer (indicating a lengthening of perceived duration) for the moving stimuli than for stationary stimuli.

\section{Method}

Subjects. Fifty-nine students ( 16 males, 43 females) from general psychology classes at the University of Southern Maine served as volunteer subjects. The students received extra course credit for their participation.

Apparatus and Stimuli. An Apple II-GS computer and 12-in. $(30.5-\mathrm{cm})$ monitor (diagonal measurement) were used to present stimuli and record responses. Events were timed with a Timemaster II H. O. clock card (Applied Engineering) set at an interrupt rate of $1024 \mathrm{~Hz}$. The computer was programmed in Applesoft Basic to present a series of high-resolution visual displays for time judgments. The displays, generated via the Graphics Magician animation software package (Penguin Software), consisted of one, three, or five white, boxlike shapes whose sides measured $5 \mathrm{~mm}$, with a $3-\mathrm{mm}$ prong extending from each corner. The stimuli were presented against a black background at various points along a $162-\mathrm{cm}$ continuous invisible pathway on the computer screen. The pathway consisted of numerous loops, curves, turns, and changes in direction. For the stationary displays, stimuli appeared at quasirandom positions along the pathway, with the restriction that the stimuli were separated and did not overlap. For the moving displays, the stimuli rotated as they traveled smoothly along the pathway at a speed of $20 \mathrm{~cm} / \mathrm{sec}$. The starting positions on each trial were selected on a quasi-random basis.

Design and Procedure. Subjects were tested individually in each condition of a $2 \times 3 \times 5$ within-groups factorial design. The factors were display (stationary or moving), target (one, three, or five stimulus figures in each display), and duration $(6,9,12,15$, or $18 \mathrm{sec})$. The resulting 30 trials were presented in an individually randomized order to each subject. Each of the trials, which were self paced, started with the message "Ready-press the spacebar" on the screen. When the spacebar on the keyboard was pressed, the stimulus display appeared, bounded at the beginning and end by a 1 -sec blank interval. Immediately following stimulus presentation, a message instructed the subject to reproduce the duration of the display by pressing the spacebar to mark the beginning and end points of his or her estimate. The testing procedure took approximately $30 \mathrm{~min}$.

\section{Results and Discussion}

The temporal reproductions were transformed into constant error scores. These values are given by the expression $(R / T)$, where $R$ corresponds to the subject's reproduction and $T$ represents the actual physical duration of the interval (see Hornstein \& Rotter, 1969). Constant error scores thus indicate whether an interval was overestimated (a value greater than 1.00) or underestimated (a value less than 1.00). Because the time estimates are expressed as proportions of physical durations, they are directly comparable across the different durations.

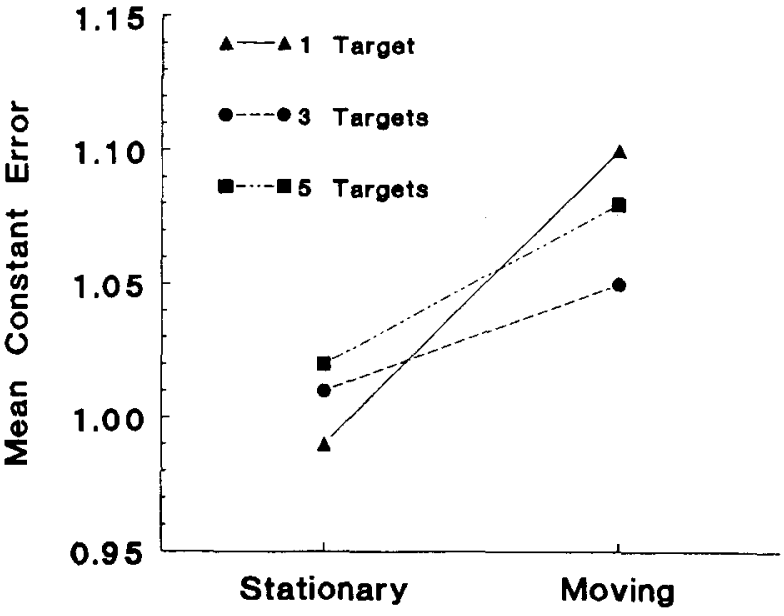

Figure 1. Mean constant error of temporal reproductions of intervals containing 1,3 , or 5 target stimuli as a function of stationary versus moving (at $20 \mathrm{~cm} / \mathrm{sec}$ ) displays in Experiment 1.

These scores were submitted to a $2 \times 3 \times 5$ repeated measures analysis of variance (ANOVA).

Display exerted a significant effect in the analysis $[F(1,58)=19.69, p<.001$; see Figure 1$]$. Subjects accurately judged the stationary displays $(M=1.01)$, but overestimated the moving displays $(M=1.08)$. The display $\times$ target interaction $[F(2,116)=3.81, p<.05]$ was probed with simple main effects tests. ${ }^{1}$ Comparisons of stationary versus moving displays showed that stimulus movement lengthened time judgments in the one-target condition $[F(1,58)=40.80, p<.001]$, the three-target condition $[F(1,58)=6.80, p<.02]$, and the five-target condition $[F(1,58)=10.80, p<.01]$. A comparison of the target conditions within each display showed that the source of the interaction involved the moving displays, with the one-target condition associated with the longest judgments $[F(2,116)=3.60, p<.05]$. The magnitude of this effect was weak, however: a relatively conservative multiple comparison test contrasting the three moving target conditions (the Tukey test; see Keppel, 1982) failed to uncover any significant differences. The only other significant result in the ANOVA was a main effect for duration $[F(4,232)=63.11, p<.001]$. The mean error scores of the 6-, 9-, 12-, 15-, and 18-sec displays were $1.20,1.11,1.03,0.96$, and 0.92 , respectively. Thus, temporal judgments were relatively long for the shorter stimulus intervals and declined in magnitude as the intervals became longer. This effect applied equally to the stationary and moving displays. A trend analysis indicated that the linear component $[F(1,58)=119.93, p<$ $.001]$ accounted for $98 \%$ of the variance.

The results show that stimulus movement alters temporal perception by lengthening apparent duration. This effect replicates some of the previous reports in the literature, and extends these findings to a procedure involving different stimuli and durations. The data also show a bias for subjects to overestimate the shorter stimulus intervals and underestimate the longer intervals, an 
effect known as Vierordt's law (Woodrow, 1951). The hypothesis that greater numbers of stimulus targets would lengthen time judgments was not supported.

\section{EXPERIMENT 2}

Experiment 2 was designed to (1) replicate the findings of Experiment 1, and (2) test the effect of stimulus speed on time judgments. Since faster speed creates more changes (i.e., more turns, variations in direction, and shifts in relative position) per unit time, faster moving stimuli should lengthen perceived time to a greater degree than those moving more slowly.

\section{Method}

Subjects. Fifty-seven students ( 23 males, 34 females) from general psychology classes volunteered as subjects, and received extra course credit for their participation.

Apparatus and Stimuli. The computer hardware was the same as that used in Experiment 1. The stimulus displays were similar also, except that in the moving displays, the stimuli traveled along the invisible pathway at either a slow $(10 \mathrm{~cm} / \mathrm{sec})$ or a fast $(30 \mathrm{~cm} / \mathrm{sec})$ speed. Note that the slow speed is slower and the faster speed faster than the $20-\mathrm{cm} / \mathrm{sec}$ rate employed in Experiment 1 .

Design and Procedure. Subjects were tested in a $3 \times 3 \times 5$ within-groups factorial design. The factors were speed $(0,10$, or $30 \mathrm{~cm} / \mathrm{sec}$ ), target (one, three, or five stimulus figures in each display), and duration $(6,9,12,15$, or $18 \mathrm{sec})$. Each subject was tested once in each of the 45 stimulus conditions. The procedure was identical to that of Experiment 1 - each self-initiated trial involved the presentation of a stimulus display, after which the subject reproduced the duration of the display. The testing procedure took approximately $45 \mathrm{~min}$.

\section{Results and Discussion}

The temporal reproductions were converted into constant error scores. These values were submitted to a $3 \times$ $3 \times 5$ repeated measures ANOVA. In addition to the standard analysis, the effect of speed was probed with a set of orthogonal planned comparisons: Contrast 1 (motion) compared stationary versus moving (both slow and fast) displays; Contrast 2 (speed) compared the slow $(10 \mathrm{~cm} / \mathrm{sec})$ versus the fast $(30 \mathrm{~cm} / \mathrm{sec})$ displays.

The standard analysis uncovered a significant effect of speed $[F(2,112)=12.38, p<.001$; see Figure 2]. Contrast $1[F(1,56)=22.49, p<.001]$ showed that the moving displays $(M=1.12)$ were overestimated relative to the stationary displays $(M=1.04)$. This outcome replicates the results of Experiment 1 in showing that stimulus motion lengthens perceived time. However, Contrast 2 (slow vs. fast) was not significant $(F<1)$. The only other significant effect in the analysis was that of duration $[F(4,224)=72.97, p<.001]$. The mean error scores of the 6-, 9-, 12-, 15-, and 18-sec displays were $1.29,1.17,1.07,0.98$, and 0.93 , respectively. This effect applied to all three speed conditions. The trend of shorter temporal judgments associated with longer durations was linear $[F(1,56)=110.20, p<.001]$, accounting for $98.9 \%$ of the variance.

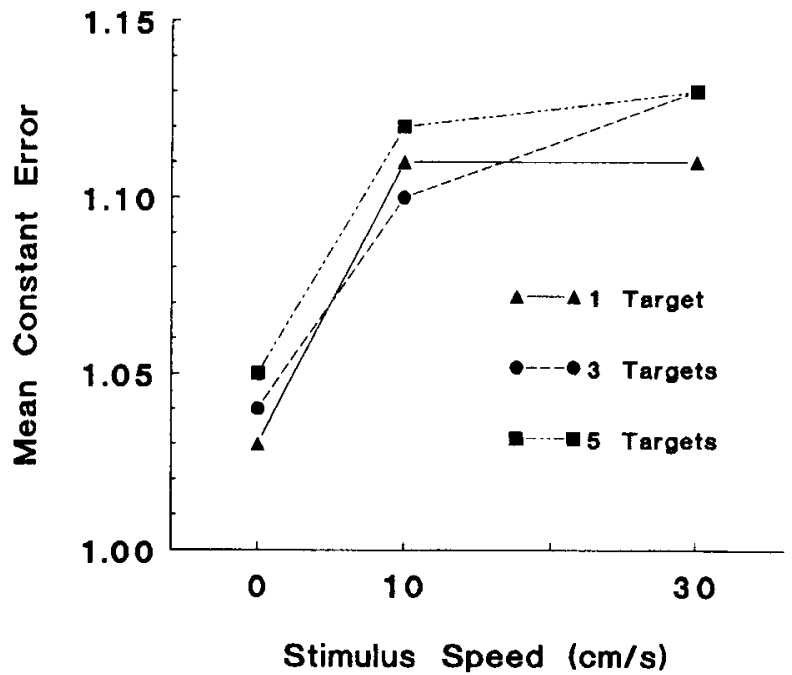

Figure 2. Mean constant error of temporal reproductions of intervals containing 1,3 , or 5 target stimuli as a function of stimulus speed in Experiment 2.

The results resemble those of Experiment 1. In both studies, moving stimuli were judged to last for a longer time than stationary stimuli, an outcome consistent with event-based models of timing. However, the predicted effect for stimulus speed was not borne out. The constant error scores for the two types of moving displays are virtually identical ( $M=1.11$ for slow displays; $M=$ 1.12 for fast displays). Both experiments also showed that shorter intervals were overestimated, while longer intervals were underestimated. The number of stimulus targets in the displays had no effect $(F<1)$.

\section{EXPERIMENT 3}

Experiment 3 was designed to determine whether the effects of stimulus motion can be demonstrated with a different time-judgment method-namely, that of temporal production. In the production task, the subject is asked to generate a specified interval, typically by activating some device such as a light or tone for the requisite duration. The production method involves an ongoing assessment of time made as the interval is presented, and as such contrasts with the method of reproduction, which requires the subject to judge an interval that has already elapsed. In terms of temporal experience, production bears an inverse relationship to reproduction; that is, underestimations with temporal production are equivalent subjectively to overestimations with reproduction, and vice versa. For example, a 5 -sec production estimate of a 10 -sec standard interval is subjectively similar to a $20-\mathrm{sec}$ reproduction of the same standard. In each case, the standard interval appears to the observer to flow at a relatively slow rate, creating a perceived lengthening of time (for comprehensive discussions of these issues, consult Bindra \& 
Waksberg, 1956; Carlson \& Feinberg, 1968; Doob, 1971, pp. 37-46; McConchie \& Rutschmann, 1971). Thus, to be consistent with the previous experiments, subjects should produce shorter intervals in response to the moving stimulus displays.

\section{Method}

Subjects. Sixty-two students ( 25 males, 37 females) in general psychology earned extra course credit for their participation.

Apparatus and Stimuli. The apparatus and stimuli were the same as those used previously. The program that controlled Experiment 2 was rewritten and modified to monitor temporal production responses rather than reproductions.

Design and Procedure. As in Experiment 2, subjects were tested individually in all conditions of a $3 \times 3 \times 5$ (speed $\times$ target $\times$ duration) within-groups design. The conditions were presented in an individually randomized sequence for each subject. Each trial began with a "Ready" message on the screen instructing the subject to press the spacebar. The ready message also contained the instruction "Time the next display for $T$ sec," where $T$ corresponds to one of five duration values $(6,9,12,15$, or $18 \mathrm{sec})$. The subject's task was to allow each display to remain on the screen for the specif ied duration by controlling its onset and offset with the spacebar. The display screen also contained a line of text at the bottom ("Display time $=T \mathrm{sec}$ "), reminding the subject how long the interval should last. The testing procedure took about $45 \mathrm{~min}$.

\section{Results and Discussion}

The constant error scores were submitted to a speed $X$ target $X$ duration repeated measures ANOVA, and the main effect for speed was significant $[F(2,122)=6.73$, $p<.002$; see Figure 3]. This effect was probed with the same orthogonal contrasts as used previously. Contrast 1 $[F(1,61)=6.16, p<.02]$ revealed that the moving displays are characterized by shorter temporal productions $(M=1.11)$ than are the stationary displays $(M=1.15)$. This result is in the predicted direction, given the inverse relationship between temporal productions and repro-

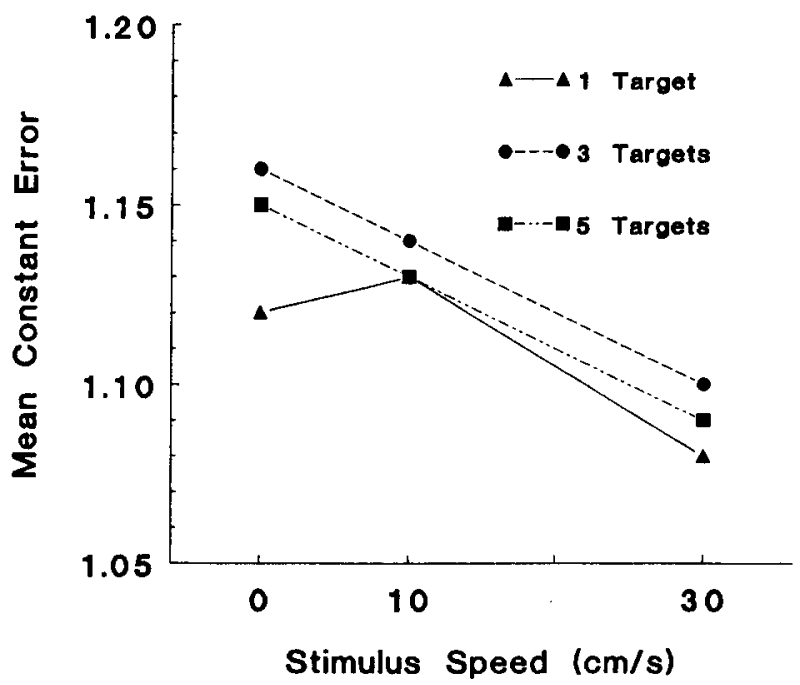

Figure 3. Mean constant error of temporal productions of intervals containing 1,3 , or 5 target stimuli as a function of stimulus speed in Experiment 3. ductions. Contrast 2 (slow vs. fast displays) was also significant $[F(1,61)=7.31, p<.01]$. The fast $(30 \mathrm{~cm} / \mathrm{sec})$ displays $(M=1.09)$ are associated with shorter productions than are the slow $(10 \mathrm{~cm} / \mathrm{sec})$ displays $(M=1.13)$. The main effect for duration $[F(4,244)=3.42, p<.01]$ was similar to that observed in the previous experiments, with shorter intervals being overestimated and longer intervals underestimated: the mean error scores of the 6-, 9-, 12-, 15-, and 18-sec displays were $1.16,1.14$, $1.12,1.09$, and 1.09 , respectively. The linear component of the trend analysis $[F(1,61)=6.34, p<.02]$ accounted for $91.6 \%$ of the variance. However, the target $\times$ duration interaction $[F(8,488)=2.81, p<.005]$ indicates that the duration main effect should be interpreted with some qualification. The interaction was analyzed with simple main effects tests, which showed that longer durations were associated with shorter judgments in both the onetarget $[F(4,244)=5.75, p<.001]$ and the three-target $[F(4,244)=4.75, p<.001]$ conditions. The effect was not significant in the five-target condition $(p<.10)$.

The only other significant effect involving targets was the speed $\times$ target $\times$ duration interaction $[F(16,976)=$ $1.73, p<.05]$. This interaction was subjected to a series of analytical comparisons. Tests of simple interaction effects (Keppel, 1982) revealed that a speed $\times$ target effect was present only in the 6 -sec duration $[F(4,244)=$ $2.56, p<.05]$. Simple, simple main effects tests contrasting target conditions within the three speeds indicated that the effect was restricted to the stationary displays $[F(2,122)=5.33, p<.01]$. Tukey multiple comparison tests showed that the three-target condition $(M=$ 1.31) was associated with longer temporal productions than were the one-target $(M=1.14, p<.01)$ and five-target $(M=1.18, p<.05)$ conditions. None of the other effects in the ANOVA were significant.

As with the reproduction data of Experiments 1 and 2, the temporal productions indicate that moving stimuli lengthened perceived time. In contrast to the results of Experiment 2, however, these results support the idea that faster movement enhances the temporal lengthening effect. The temporal productions also exhibited the standard Vierordt's law pattern of longer stimulus durations associated with shorter time judgments. Moderate numbers of targets were related to longer temporal productions, but only for the 6-sec stationary displays. A similar pattern has been observed elsewhere (Poynter \& Homa, 1983), and this effect will be considered in more detail in the general discussion section.

\section{EXPERIMENT 4}

Experiment 4 more closely examined the effect of stimulus speed on perceived time. Although event-based models predict that faster speeds should lengthen time judgments to a greater degree than slower speeds, the literature on this issue is contradictory. The present research has also produced mixed results, with Experiment 2 showing no effect for speed but Experiment 3 
exhibiting the predicted effect. One possible explanation-that stimulus speed was not adequately manipulated, and that a broader range of speed conditions may be necessary to produce reliable differences-was tested by exposing subjects to a larger set of stimulus speeds than were used previously.

\section{Method}

Subjects. Ninety-one students ( 30 males, 61 females) from general psychology classes served as subjects and received extra course credit for their participation.

Apparatus and Stimuli. The computer hardware was the same as that used in the previous experiments. Each display for time judgments consisted of three white cross-shaped stimulus forms measuring $6 \mathrm{~mm}$ on each diagonal. These stimuli were presented against a black background at various points along a $243-\mathrm{cm}$ continuous invisible pathway on the monitor screen. As before, the pathway consisted of numerous loops, curves, and turns. The stimuli in each display were either stationary or moving at speeds ranging from 5 to $45 \mathrm{~cm} / \mathrm{sec}$. In the stationary displays, the stimuli were plotted in static positions at quasi-random points along the pathway; in the moving displays, the stimuli rotated as they traveled smoothly along the pathway.

Design and Procedure. Subjects were tested individually in each condition of a $6 \times 5$ within-groups factorial design. The factors were speed $(0,5,15,25,35$, or $45 \mathrm{~cm} / \mathrm{sec})$ and duration $(6,9,12$, 15 , or $18 \mathrm{sec})$. The resulting 30 self-initiated trials were presented to each subject in an individually randomized order; the subject reproduced the duration of each display. The testing session lasted about $30 \mathrm{~min}$.

\section{Results and Discussion}

The constant error scores were submitted to a $6 \times 5$ repeated measures ANOVA, and each of the effects was significant. The main effect for speed $[F(5,450)=2.88$, $p<.01]$ is depicted in Figure 4. An a priori comparison contrasting judgments of the stationary versus the moving stimuli replicated the previous findings; that is, moving displays $(M=1.05)$ were judged to be longer in duration than were stationary displays $[M=1.02 ; F(1,90)=$
$3.87, p<.05]$. A trend analysis (adjusted for the unequal spacing of stimulus speeds) was also performed, and the linear component was significant $[F(1,90)=12.68, p<$ $.001]$. The linear increase in judged time as a function of stimulus speed accounted for $93.5 \%$ of the variance. The main effect for duration $[F(4,360)=75.90, p<.001]$ showed the same pattern as before, with short intervals being overestimated and long intervals underestimated. The mean error scores for the 6-, 9-, 12-, 15-, and 18-sec displays were $1.22,1.10,1.03,0.98$, and 0.90 , respectively. A trend analysis of these data indicated that the linear component $[F(1,90)=167.93, p<.001]$ accounted for $97.5 \%$ of the variance.

The speed $\times$ duration interaction $[F(20,1800)=2.97$, $p<.001]$ was probed with simple main effects tests contrasting stimulus speeds within the different durations. These tests showed that the most reliable differences occurred in the shorter (6-and 9-sec) durations. In the 6-sec condition $[F(5,450)=7.17, p<.001]$, the mean error scores for the 0-, 5-, 15-, 25-, 35-, and 45-cm/sec speeds were $1.14,1.16,1.26,1.19,1.32$, and 1.23 , respectively. These data show that the stationary displays $(M=1.14)$ yielded the shortest judgments, whereas the overall combined mean of the moving displays $(M=1.23)$ represents a $7.9 \%$ lengthening of judged time. A similar outcome occurred in the 9-sec condition $[F(5,450)=4.33, p<$ $.001]$, where the mean error scores for the 0-, 5-, 15-, $25-, 35-$, and $45-\mathrm{cm} / \mathrm{sec}$ speeds were $1.02,1.12,1.08$, $1.17,1.07$, and 1.13 , respectively. The shortest (and most accurate) time judgments were associated with the stationary displays $(M=1.02)$, while the moving displays (overall $M=1.11$ ) lengthened judgments by $8.8 \%$.

The main effect for speed indicates that faster stimulus motion leads to progressively longer temporal judgments, and that this increase is linear. This result supports the predictions of a change model-namely, that faster movements produce more changes, and more

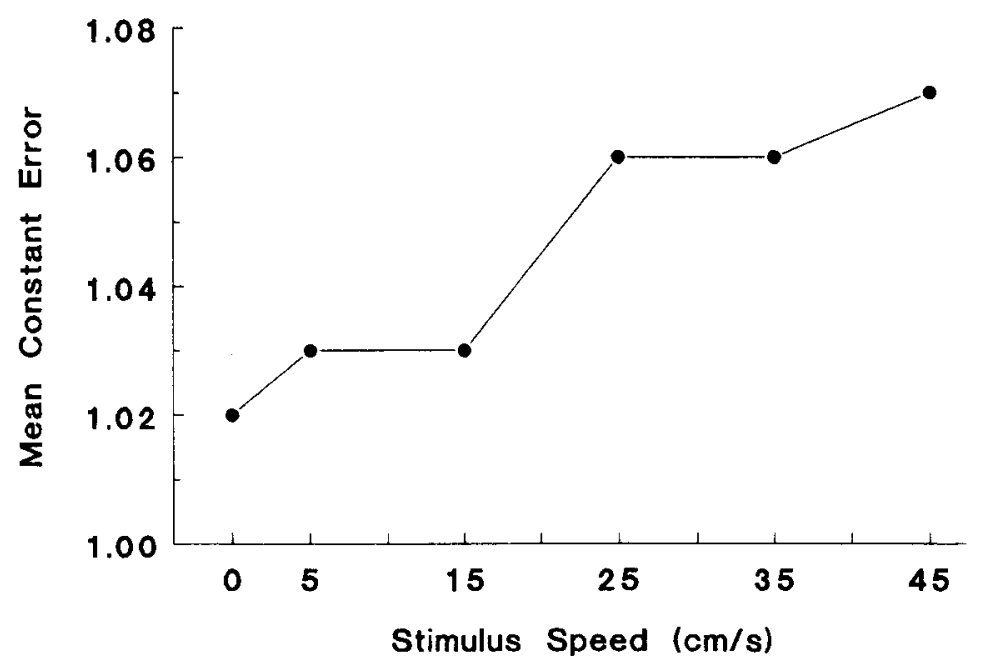

Figure 4. Mean constant error of temporal reproductions as a function of stimulus speed in Experiment 4. 
changes lengthen perceived time. The finding that the temporal lengthening effect was stronger for the stimuli of shorter duration replicates findings reported by Tayama and Aiba (1982). The results also show that subjects tended to overestimate shorter intervals and underestimate longer intervals - an effect which occurred across all the stimulus speeds tested.

\section{EXPERIMENT 5}

Experiment 5 served two purposes, the first of which was to replicate the progressive temporal lengthening effect of stimulus speed. A smaller number of speeds was employed than in Experiment 4, but they covered the same $0-45 \mathrm{~cm} / \mathrm{sec}$ range. The second purpose was to investigate in more detail the role of the number of stimulus elements comprising the displays. In Experiments 1, 2 , and 3 , variation in the number of stimulus targets (from 1 to 5 ) had little or no impact on time judgments. This result is consistent with the predictions of the change/segmentation model and contradicts the storagesize model. However, it could be argued that the differences between target conditions were too small, and that a stronger manipulation of this variable may have produced an effect. This possibility was checked by exaggerating the difference in the number of stimuli appearing in the displays.

\section{Method}

Subjects. One-hundred fourteen students ( 45 males, 69 females) from general psychology classes volunteered as subjects in exchange for extra course credit.

Apparatus and Stimuli. The computer hardware was the same as that used in the previous experiments. The stimulus forms in each display were nonrotating squares whose sides each measured $6 \mathrm{~mm}$; these stimuli moved continuously along a complex $372-\mathrm{cm}$ invisible pathway. Half of the displays contained single stimuli and half contained multiple stimuli. The single displays consisted of three separate stimulus targets located at points separated by an equal distance along the pathway. The multiple displays consisted of three groupings of stimuli, with each group composed of a linear chain of 35 partially overlapping individual squares separated from one another by $5 \mathrm{~mm}$. Hence, each display contained either 3 or 105 target stimuli.

Design and Procedure. Subjects were tested in all conditions of a $2 \times 4 \times 5$ within-groups design. The factors were target (single or multiple), speed $(0,5,25$, or $45 \mathrm{~cm} / \mathrm{sec})$, and duration $(6,9,12$, 15 , or $18 \mathrm{sec}$ ). The procedure was the same as that used before; that is, each self-initiated trial consisted of the presentation of a stimulus display, after which the subject was prompted to reproduce the duration of the display. Each subject was tested once in each of the 40 conditions, and each session lasted approximately $45 \mathrm{~min}$.

\section{Results and Discussion}

The temporal reproductions were converted into constant error scores and submitted to a $2 \times 4 \times 5$ repeated measures ANOVA. The main effect for speed was significant $[F(3,339)=8.42, p<.001$; see Figure 5]. This effect was first probed with a planned comparison contrasting the stationary versus the moving displays. As expected, the moving displays $(M=0.99)$ were judged

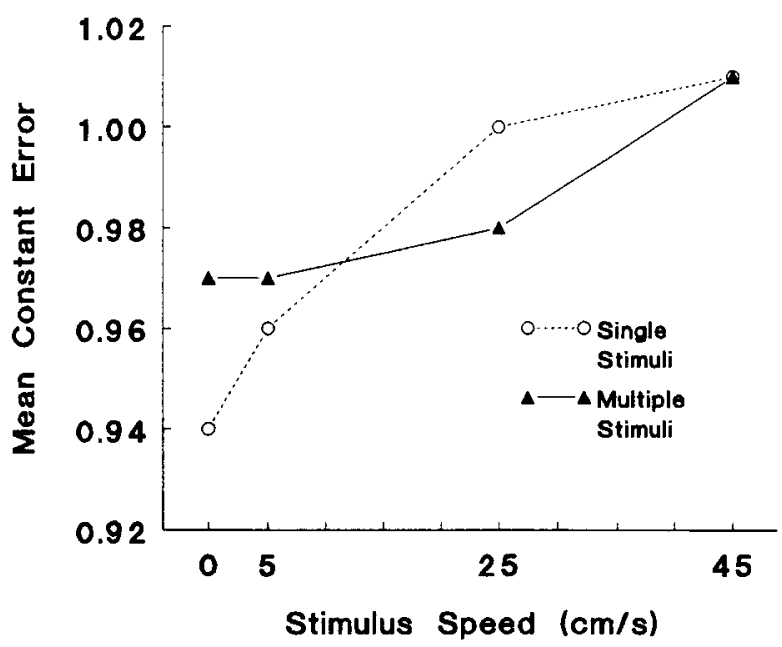

Figure 5. Mean constant error of temporal reproductions of intervals containing single- or multiple-target stimuli as a function of stimulus speed in Experiment 5.

to be longer than the stationary displays $(M=0.96)$; $F(1,113)=4.37, p<.05]$. The mean error scores for the 0 -, $5-, 25-$, and $45-\mathrm{cm} / \mathrm{sec}$ speeds were $0.96,0.96,0.99$, and 1.01 , respectively. A trend analysis of these scores (adjusted for unequal spacing of the stimulus speeds) showed that the linear component $[F(1,113)=22.92, p<.001]$ accounted for $99.9 \%$ of the variance. This result replicates those of Experiments 3 and 4, demonstrating that faster stimulus movement lengthens perceived time to a greater degree than slower stimulus movement. Duration also exerted a significant main effect $[F(4,452)=125.36, p<$ $.001]$. The mean error scores for the 6-, 9-, 12-, 15-, and 18 -sec displays were $1.11,1.04,0.97,0.90$, and 0.85 , respectively. The trend of shorter temporal judgments associated with longer durations was linear $[F(1,113)=$ $258.97, p<.001]$, accounting for $98.7 \%$ of the variance.

The speed $\times$ duration interaction $[F(12,1356)=3.60$, $p<.001]$ was analyzed with simple main effects tests comparing stimulus speeds within each duration. These comparisons revealed that the temporal lengthening effect of stimulus speed occurred mainly in the 6-sec $[F(3,339)=14.50, p<.001], 9-\sec [F(3,339)=5.00, p<$ $.01]$, and $12-\sec [F(3,339)=3.46, p<.02]$ conditions. In the 6-sec condition, the mean error scores for the 0-, 5-, $25-$, and $45-\mathrm{cm} / \mathrm{sec}$ speeds were $1.06,1.12,1.15$, and 1.21 , respectively. A comparison of the stationary versus the combined moving displays shows that stimulus motion lengthened time judgments by $9.4 \%$. In the $9-\mathrm{sec}$ condition, the mean error scores for the four speeds were $1.02,1.02,1.03$, and 1.09; stimulus motion produced an average lengthening in time judgments of $2.9 \%$. In the 12 -sec condition, the mean error scores for the four speeds were $0.94,0.95,1.01$, and 0.98 . The stationaryversus-moving comparison shows a $4.3 \%$ temporal lengthening effect of motion.

The only significant effect involving target was the 3way interaction $[F(12,1356)=2.54, p<.01]$. This inter- 
action was probed with a set of analytical contrasts. An analysis of simple interaction effects examined the influence of targets and durations within the different speed conditions, and showed that a significant target $X$ duration interaction was confined to the stationary speed condition $[F(4,452)=4.17, p<.01]$. Tests of simple, simple main effects contrasting the two types of target displays within each duration revealed that significant differences occurred only for stimuli presented for $6 \mathrm{sec}$ $[F(1,113)=14.67, p<.001]$. Subjects in this case were accurate in judging the single stimuli $(M=1.00)$, but overestimated the multiple stimuli $(M=1.12)$.

As in the previous experiments, stimulus motion lengthened perceived time. The results also showed that faster speeds are associated with longer time judgments, particularly for the stimuli of shorter duration. The role of the number of stimulus targets was limited; greater numbers of stimuli lengthened time judgments, but only in the short-duration stationary displays. These data suggest that stimulus motion is a more important temporal cue than stimulus number. The duration main effect showed the same pattern of decreasing time estimates as a function of increasing display durations that was observed in each of the previous experiments.

\section{GENERAL DISCUSSION}

Five experiments were conducted to assess the effects of stimulus motion on perceived time. The influence of different speeds, durations, and numbers of stimuli was also examined. The experiments produced a consistent pattern of results: (1) stimulus motion lengthened perceived time; (2) faster speeds generally lengthened perceived time to a greater degree than slower speeds; (3) the time judgments for both stationary and moving stimuli conformed to Vierordt's law; and (4) the number of stimulus elements had only a limited influence on time judgments. The results can be accommodated within a theoretical framework emphasizing the role of stimulus changes in time perception.

A consistent finding was that moving stimuli were judged to last for a longer duration than were stationary stimuli. This result occurred in each of the five experiments, and replicates the findings of some earlier reports (Goldstone \& Lhamon, 1974; Lhamon \& Goldstone, 1975; Roelofs \& Zeeman, 1951; Tayama \& Aiba, 1982). Experiment 3 is of particular interest, as the results correspond to the inverse relation between the methods of reproduction and production. The longer temporal reproductions (Experiments 1, 2, 4, and 5) and the shorter temporal productions (Experiment 3 ) associated with moving displays both show that stimulus motion lengthened perceived time. These results parallel the temporal production data reported by Tayama et al. (1987, Experiment 2). This temporal lengthening effect is in line with a basic assumption shared by many theorists - namely, that the perception of time is ultimately the perception of stimulus changes. Michon's (1972, 1985; Michon \& Jackson, 1984) concept of "temporal information" is representative of this assumption. Michon maintains that our experience of time depends upon the amount of temporal information present during an interval, and that a perceived sequence of events (i.e., order, succession, and change) constitutes temporal information. Moving objects, by virtue of possessing more temporal information in the form of changing spatial locations, would be expected to lengthen temporal judgments. From an ecological viewpoint (Gibson, 1975), movement is a primary source of temporal information because it is one of the basic "natural events in the terrestrial world, where pebbles roll, the leaves fall, the streams flow, and animals scurry about" (p. 297). In and of itself, the effect of stimulus motion could be explained by other event-based theories of time perception. The storage-size model would argue that moving stimuli represent a more complex pattern of events than stationary stimuli, and hence lengthen perceived time because they require more memory space. It is clear that the change/segmentation and storage-size models of ten lead to similar predictions and may explain the same set of experimental findings (see Poynter, 1989, p. 307). However, the results involving stimulus targets (see below) are consistent with the change/segmentation model, but provide only limited support for a storagesize interpretation.

While the earlier literature concerning stimulus speed has produced inconclusive results, the present research has produced several important findings on this topic. Stimulus speed was varied in four experiments (Experiments 2, 3, 4, and 5), three of which showed the predicted effect of faster speeds associated with longer time judgments. This outcome replicates some previous findings (e.g., Tayama \& Aiba, 1982; Tayama et al., 1987), and directly contradicts a reported negative relation between speed and perceived time (the i' effect of Matsuda, 1974). Indeed, Experiments 4 and 5 demonstrate a positive linear relation between stimulus speed and time judgments which accounts for well over $90 \%$ of the variance. This research also offers a possible explanation as to why speed effects may not always be observed: the influence of speed may be moderated by stimulus duration. Experiments 4 and 5 both uncovered speed $\times$ duration interactions showing that speed exerted less influence on the relatively longer (15-and 18-sec) stimulus displays. This finding corresponds to a similar result reported by Tayama and Aiba (1982, Experiment 2). The effect is also consistent with Poynter's (1989) notion that different cues, strategies, and timing mechanisms vary in importance across different contexts. In addition, the speed $\times$ duration interactions discount a simple "distance traveled" hypothesis as an explanation of the results. If time judgments are based primarily on the physical distance an object travels (such that the longer the distance, the greater the lengthening of perceived time), then the longest judgments should be associated with the stimuli that are the fastest and of the longest duration. The fact that such a result did not occur argues against the hypothesis. 
Vierordt's law was observed in each experiment. Vierordt's law-a tendency to overestimate the shorter intervals in a series and underestimate the longer intervals - is regarded as the oldest finding in time psychology (see Boring, 1942; Woodrow, 1951). This effect may be understood in terms of a tendency for perceptual judgments to regress from extreme values to the midpoint of the stimulus range (Bobko, Schiffman, Castino, \& Chiappetta, 1977; Helson, 1964). The important point here is that Vierordt's law applied equally to judgments of both stationary and moving displays, despite the fact that, overall, moving stimuli were judged to be of longer duration than stationary stimuli. This result indicates that Vierordt's law is a robust phenomenon, unaffected by the presence of other stimulus factors relevant to timing. One other point to note is that the same pattern of decreasing judgments associated with increasing durations occurred with both temporal reproductions (Experiments 1, 2, 4, and 5) and temporal productions (Experiment 3 ), despite the inverse relation between the two types of time judgments. This result is not unusual, however. Although most studies of Vierordt's law involve reproduction (Bobko et al., 1977; Schiffman \& Bobko, 1974, 1977; Woodrow, 1934), the same pattern has been found in various experiments involving temporal production (e.g., Brown \& West, 1990; Clausen, 1950; Doehring, 1961; ${ }^{2}$ Hawkes, Bailey, \& Warm, 1961; Spivack \& Levine, 1964). Vierordt's law appears to be a general response bias effect that occurs under a wide variety of experimental conditions.

The number of stimulus targets did not produce a main effect in any of the experiments, and neither was there a simple speed $\times$ target interaction, which would result if target effects occurred only in the stationary displays. These findings run counter to the predictions of the storage-size model and the associated filled-duration illusion. The status of the filled-duration illusion has already been questioned; both Doob (1971, pp. 115-121) and Wallace and Rabin (1960) report many inconsistencies and null results in their reviews of the classical literature on the topic. More recent investigations indicate that the presence and strength of the illusion is affected by a variety of factors, including the duration of the stimuli (Ihle \& Wilsoncroft, 1983), attentional processes (Adams, 1977), and context effects (Gomez \& Robertson, 1979; Robertson \& Gomez, 1980). An example of this complexity was observed in Experiment 3 . The effect, restricted to the 6-sec stationary displays, showed that temporal productions were greatest for a medium number of targets. The mean temporal production scores for the 1-, 3-, and 5-target conditions were 1.14, 1.31, and 1.18 , respectively. This result does not fit the standard pattern of the filled-duration illusion. However, the result is consistent with a model describing the relationship between stimulus events and duration proposed by Poynter (1989, pp. 315-316; Poynter \& Homa, 1983, p. 558). In this model, increasing numbers of stimuli lengthen perceived time for short durations (e.g., $2 \mathrm{sec}$ ), shorten perceived time for long durations $(16 \mathrm{sec}$ or greater), and produce a U-shaped function for intermediate durations. The temporal productions fit this intermediate pattern (given that short productions represent a lengthening of perceived time, and long productions correspond to a shortening of perceived time).

Other evidence indicates that stimulus change may be a crucial factor underlying the filled-duration illusion. In a careful multiple-experiment investigation, Poynter and Homa (1983) manipulated the degree of change while holding constant the number and complexity of stimulus events, and found that more changes led to a greater lengthening of perceived time. This study is notable for controlling different stimulus characteristics, allowing one to gauge the relative contribution of these factors in timing. As Poynter and Homa's results suggest, stimulus factors may differ in their degree of importance in timing. If subjects are exposed to intervals that vary only in the number or size of stimulus elements, then these factors may influence time judgments. If, however, the intervals contain a richer set of temporal cues in the form of changing stimulus events, the effects of number or size may diminish in importance. In the present research, the relatively weak effects of stimulus number were superseded by the effects of stimulus motion. Experiment 5 shows a shift in the salience of these temporal cues. Short-duration (6-sec) displays containing large numbers of stimuli $(n=105)$ were judged to be longer than corresponding displays containing small numbers of stimuli $(n=3$; i.e., the filledduration illusion), but the effect occurred only when the stimuli were stationary. When the stimuli were in motion, the illusion disappeared and temporal judgments were influenced by stimulus speed and duration. This finding is in line with the idea that various temporal cues are differentially effective across different situations, an idea regarded by Poynter $(1989$, p. 309) as important in distinguishing the change/segmentation model from other theories of timing.

The results point to several directions for future research. First, manipulating the manner in which stimuli move may provide alternative ways to test the change model. For example, one could compare time judgments of stimuli that move in a smooth, continuous manner (as in the present case) with those that move in an incremental, stop-and-go fashion by jumping from point to point. Incremental movement produces more abrupt, discrete changes, which may enhance the temporal lengthening effect of motion.

Second, manipulations of the stimuli themselves may allow for a direct comparison of the change/segmentation and storage-size models. In the present investigation, multiple stimuli were designed to be similar and indistinguishable (they all had the same shape, speed, trajectory, etc.). An alternative design might independently vary both the distinctiveness and the number of stimuli; in one condition, stimuli would be identical in appearance and movement, while in another condition, they would be distinctive and would move along unique trajectories. According to the storage-size model, the 
filled-duration illusion would occur in both conditions; according to the change/segmentation model, however, the illusion would occur only in the distinctive condition.

A third research area involves the relationship between speed and duration. The progressive temporal lengthening effect associated with faster speeds applies mainly to shorter durations. It would be valuable to systematically explore how this transition in the effectiveness of speed (and other temporal cues) is affected by variations in the magnitude of duration. Finally, the concept of change has a great deal of ecological relevance for the psychology of time (Gibson, 1975). Given that change is a fundamental property of the natural environment, it is perhaps not surprising that timing is attuned to, and affected by, the motion and speed of moving objects. One challenge, however, is to specify change more precisely. As Poynter (1989) argues, change involves more than simply a succession of events. The perceived organization, salience, and distinctiveness of these events are also important.

\section{REFERENCES}

Adams, R. D. (1977). Intervening stimulus effects on category judgments of duration. Perception \& Psychophysics, 21, 527-534.

ALGOM, D., \& COHEN-RAZ, L. (1984). Visual velocity input-output functions: The integration of distance and duration onto subjective velocity. Journal of Experimental Psychology: Human Perception \& Performance, 10, 486-501.

BindRA, D., \& WAKSBERG, H. (1956). Methods and terminology in studies of time estimation. Psychological Bulletin, 53, 155-159.

BLOCK, R. A. (1978). Remembered duration: Effects of event and sequence complexity. Memory \& Cognition, 6, 320-326.

BLOCK, R. A. (1982). Temporal judgments and contextual change. Journal of Experimental Psychology: Learning, Memory, \& Cognition, 8, 530-544.

Block, R. A. (1990). Models of psychological time. In R. A. Block (Ed.), Cognitive models of psychological time (pp. 1-35). Hillsdale, $\mathrm{NJ}$ : Erlbaum.

Bobko, D. J., Schiffman, H. R., Castino, R. J., \& Chiappetta, W. (1977). Contextual effects in duration experience. American Journal of Psychology, 90, 577-586.

BONNET, C. (1965). Influence de la vitesse du mouvement et de l'espace parcounu sur l'estimation du temps [Influence of speed of movement and of space traversed on time estimation]. Année Psychologique, 65, 357-363.

BONNET, C. (1967). Influence de la vitesse du mouvement et de l'espace parcouru sur l'estimation du temps: II [Influence of speed of movement and of space traversed on time estimation: II]. Annee Psychologique, 67, 51-60.

BONNET, C. (1968). Le rôle des changements continus et discontinus dans l'estimation de la durée d'un mouvement [The role of continuous and discontinuous change in the estimation of the duration of a movement]. Année Psychologique, 68, 347-356.

Boring, E. G. (1942). Sensation and perception in the history of experimental psychology. New York: Appleton-Century-Crofts.

Brown, J. F. (1931). On time perception in visual movement fields. Psychologische Forschung, 14, 233-248.

Brown, S. W., \& West, A. N. (1990). Multiple timing and the allocation of attention. Acta Psychologica, 75, 103-121.

Carlson, V. R., \& FeinberG, I. (1968). Individual variations in time judgment and the concept of an internal clock. Journal of Experimental Psychology, 77, 631-640.

Cavallo, V., \& Laurent, M. (1988). Visual information and skill level in time-to-collision estimation. Perception, 17, 623-632.

Clausen, J. (1950). An evaluation of experimental methods of time judgment. Journal of Experimental Psychology, 40, 756-762.
DOEHRING, D. G. (1961). Accuracy and consistency of time-estimation by four methods of reproduction. American Journal of Psychology, 74, 27-35.

Doов, L. W. (1971). Patterning of time. New Haven: Yale University Press.

Fraisse, P. (1962). Influence de la vitesse des mouvements sur l'estimation de leur durée [Influence of the speed of movement on the estimation of its duration]. Année Psychologique, 62, 391-399.

Fraisse, P. (1963). The psychology of time. New York: Harper and Row.

FraisSE, P. (1978). Time and rhythm perception. In E. C. Carterette \& M. P. Friedman (Eds.), Handbook of perception: Vol. VIII. Perceptual coding (pp. 203-254). New York: Academic Press.

Gibson, J. J. (1975). Events are perceivable but time is not. In J. T. Fraser \& N. Lawrence (Eds.), The study of time II (pp. 295-301). New York: Springer-Verlag.

GoldSTONE, S., \& Lhamon, W. T. (1974). Studies of auditory-visual differences in human time judgment: 1 . Sounds are judged longer than lights. Perceptual \& Motor Skills, 39, 63-82.

Gomez, L. M., \& RoserTSON, L. C. (1979). The filled-duration illusion: The function of temporal and nontemporal set. Perception \& Psychophysics, 25, 432-438.

HaWkes, G. R., Bailey, R. W., \& WARM, J. S. (1961). Method and modality in judgments of brief stimulus duration. Journal of Auditory Research, 1,133-144.

HeLson, H. (1964). Adaptation-level theory. New York: Harper and Row.

HoRnStein, A. D., \& Rotter, G. S. (1969). Research methodology in temporal perception. Journal of Experimental Psychology, 79, 561 564.

IHLE, R. C., \& Wilsoncroft, W. E. (1983). The filled-duration illusion: Limits of duration of interval and auditory fillers. Perceptual \& Motor Skills, 56, 655-660.

JANKOWITZ, A. Z. (1977). Ornstein's "storage size" metaphor: A cautionary note. Perceptual \& Motor Skills, 45, 284-286.

KePPEL, G. (1982). Design and analysis: A researcher's handbook (2nd ed.). Englewood Cliffs, NJ: Prentice-Hall.

Lappin, J. S., Bell, H. H., Harm, O. J., \& Kottas, B. (1975). On the relation between time and space in the visual discrimination of velocity. Journal of Experimental Psychology: Human Perception \& Performance, 1, 383-394.

Leiser, D., Stern, E., \& Meyer, J. (1991). Mean velocity and total time estimation effects of order and proportions. Journal of Environmental Psychology, 11, 347-358.

Lhamon, W. T., \& Goldstone, S. (1975). Movement and the judged duration of visual targets. Bulletin of the Psychonomic Society, 5 53-54.

MATSUDA, F. (1974). Effects of space and velocity on time estimation in children and adults. Psychological Research, 37, 107-123.

MCCONCHIE, R. D., \& RutschmanN, J. (1971). Human time estimation: On differences between methods. Perceptual \& Motor Skills, 32, 319-336

Michon, J. A. (1972). Processing of temporal information and the cognitive theory of time experience. In J. T. Fraser, F. C. Haber, \& G. W. Muller (Eds.), The study of time (pp. 242-258). New York: Springer-Verlag

Michon, J. A. (1985). The compleat time experiencer. In J. A. Michon \& J. L. Jackson (Eds.), Time, mind, and behavior (pp. 20-52). New York: Springer-Verlag.

MichON, J. A., \& JACKSON, J. L. (1984). Attentional effort and cognitive strategies in the processing of temporal information. In J. Gibbon \& L. Allan (Eds.), Timing and time perception (Annals of the New York Academy of Sciences, vol. 423, pp. 298-321). New York: New York Academy of Sciences.

Mo, S. S. (1974). Comparative judgment of temporal duration in conjunction with contextual variability: A test of a memory storage model of temporal judgment. Perceptual \& Motor Skills, 38, 10311036.

Ornstein, R. E. (1969). On the experience of time. New York: Penguin Books.

Peterken, C., Brown, B., \& Bowman, K. (1991). Predicting the future position of a moving target. Perception, 20, 5-16. 
PiageT, J. (1969). The mechanisms of perception (G. N. Seagrim, Trans.). New York: Basic Books. (Original work published 1961.)

PoYNTER, W. D. (1989). Judging the duration of time intervals: A process of remembering segments of experience. In I. Levin \& D. Zakay (Eds.), Time and human cognition: A life-span perspective (pp. 305-321). Amsterdam: Elsevier.

Poynter, W. D., \& Homa, D. (1983). Duration judgment and the experience of change. Perception \& Psychophysics, 33, 548-560.

RaChLIN, H. C. (1966). Scaling subjective velocity, distance, and duration. Perception \& Psychophysics, 1, 77-82.

Robertson, L. C., \& Gomez, L. M. (1980). Figural vs. configural effects in the filled duration illusion. Perception \& Psychophysics, 27, $111-116$.

RoElofs, C. O., \& ZEEMAN, W. P. C. (1951). Influence of different sequences of optical stimuli on the estimation of duration of a given interval of time. Acta Psychologica, 8, 89-128.

SCHIFF, W., \& OLDAK, R. (1990). Accuracy of judging time to arrival: Effects of modality, trajectory, and gender. Journal of Experimental Psychology: Human Perception \& Performance, 16, 303-316.

Schiffman, H. R., \& BoBko, D. J. (1974). Effects of stimulus complexity on the perception of brief temporal intervals. Journal of Experimental Psychology, 103, 156-159.

SCHIFFMAN, H. R., \& BoBko, D. J. (1977). The role of number and familiarity of stimuli on the perception of brief temporal intervals. American Journal of Psychology, 90, 85-93.

SPivaCK, G., \& LeVine, M. (1964). Consistency of individual differences in time judgments. Perceptual \& Motor Skills, 19, 83-92.

TAYAma, T., \& AiBA, T. S. (1982). The influence of spatial attributes of stimuli upon time estimation. Hokkaido Behavioral Science Report (Series P, Supplement No. 28). (In Japanese with English abstract.)

Tayama, T., Nakamura, M., \& Aiba, T. S. (1987). Estimated duration for rotating-spot-pattern. Japanese Psychological Research, 29, 173-183.

TYNan, P. D., \& SEKULER, R. (1982). Motion processing in peripheral vision: Reaction time and perceived velocity. Vision Research, 22, $61-68$.

VAN Doorn, A. J., \& Koenderink, J. J. (1984). Spatiotemporal integration in the detection of coherent motion. Vision Research, 24, 47 53.

Verri, A., Girosi, F., \& Torre, V. (1990). Differential techniques for optical flow. Journal of the Optical Society of America, 7, 912-922.

Wallace, M., \& Rabin, A. I. (1960). Temporal experience. Psychological Bulletin, 57, 213-236.

Wertheimer, M. (1958). Principles of perceptual organization (Abridged trans. by M. Wertheimer). In D. S. Beardslee \& M. Wertheimer (Eds.), Readings in perception (pp. 115-137). New York: Van Nostrand. (Reprinted from Psychologische Forschung, 1923, 4 , 301-350.)

WOODRow, H. (1934). The temporal indifference interval determined by the method of mean error. Journal of Experimental Psychology, 17, 167-188.

Woodrow, H. (1951). Time perception. In S. S. Stevens (Ed.), Handbook of experimental psychology (pp. 1224-1236). New York: Wiley.

\section{NOTES}

1. These and all other simple effects tests reported in this paper utilize pooled error terms.

2. Doehring (1961) conducted a comparative investigation of what he called four different methods of reproduction. However, this study provides an example of the terminological difficulties that may be encountered in the time literature. Although Doehring's Methods I, II, and IV clearly involve reproduction, Method III is more properly considered to be a temporal production task.

(Manuscript received May 23, 1994; revision accepted for publication July 26, 1994.) 